\title{
Operative Therapie des Ulcus cruris
}

\section{Surgical Treatment of Leg Ulcers}

Autor

Institut

\section{A. Mechlin}

Hautklinik, Klinikum Nürnberg (Ltd. Arzt: Prof. Dr. E. S. Schultz)

\section{Bibliografie}

DOI http://dx.doi.org/ 10.1055/s-0029-1244106

Online-Publikation: 7. 4. 2010

Akt Dermatol 2010; 36:

259-267 @ Georg Thieme

Verlag KG Stuttgart · New York ISSN 0340-2541

\section{Korrespondenzadresse} Dr. med. Axel Mechlin Hautklinik

am Klinikum Nürnberg

Prof.-Ernst-Nathan-Str. 1 90419 Nürnberg mechlin@klinikumnuernberg.de

\section{Zusammenfassung \\ $\nabla$}

Das polyätiologische Krankheitsbild des Ulcus cruris stellt oft eine therapeutische Herausforderung dar. Trotz stadiengerechter Wundbehandlung tritt gerade bei lang bestehenden Ulzera nicht selten eine Therapieresistenz auf. Um diese $\mathrm{zu}$ überwinden, bedarf es einer funktionellen operativen Therapie des Ulcus cruris. Hierfür stehen mittlerweile eine Reihe von etablierten Operationsverfahren zur Verfügung, die sowohl

\section{Epidemiologie}

Das Ulcus cruris gilt in den Industrieländern als Volkskrankheit. Laut der „Bonner Venenstudie“ aus dem Jahr 2003 liegt die Prävalenz des Ulcus cruris in Deutschland bei $0,2 \%$. Diese ist stark altersabhängig. So steigt sie ab dem 70 . Lebensjahr auf 2,5\% und Frauen sind häufiger betroffen. Allein die Zahl der therapieresistenten venösen Ulzera in Deutschland wird auf über 25000 geschätzt [7,12]. Volkswirtschaftlich betrachtet, verursacht das Ulcus cruris jährliche Kosten von ca. einer Milliarde Euro allein im Bundesgebiet.

\section{Ätiopathogenese}

Die Ursachen, die einem Ulcus cruris zugrunde liegen, sind zahlreich und zum Teil sehr unterschiedlich. Im Vordergrund stehen jedoch vaskuläre Ursachen, wobei in erster Linie die chronisch venöse Insuffizienz (CVI) und die periphere arterielle Verschlusskrankheit (pAVK) zu nennen sind. Je nach Studie oder Untersuchung lassen sich ca. $90 \%$ aller Ulcera crurum auf diese beiden Gefäßerkrankungen zurückführen. Annäherungsweise kann man davon ausgehen, dass $70 \%$ venös (Ulcus symptomatisch als auch kausal zielführend sind. Entscheidend für den Erfolg der Behandlung ist die richtige Indikationsstellung sowie die optimale Auswahl und Kombination der Operationstechniken, wobei die Berücksichtigung der ätiologisch zugrunde liegenden Ursachen wesentlich ist. Eine herausragende Bedeutung für den dauerhaften Therapieerfolg nach einer operativen Behandlung hat eine konsequente Nachbehandlung, die sich ebenfalls am Grundleiden orientiert.

cruris venosum), $10 \%$ arteriell (Ulcus arteriosum), $10 \%$ kombiniert venös und arteriell (Ulcus cruris mixtum) und weitere $10 \%$ andersartig bedingt sind. Die häufigste Ursache des Ulcus cruris ist die CVI. Sie resultiert aus der primären Varicosis oder dem postthrombotischen Syndrom (PTS).

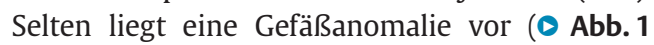
und 2).

\section{Das therapieresistente Ulcus cruris $\nabla$}

Entsprechend den Leitlinien zur Diagnostik und Therapie des Ulcus cruris venosum der Deutschen Gesellschaft für Phlebologie gilt ein Ulcus cruris als therapieresistent, wenn es unter optimaler Therapie mit Ausschöpfung aller konservativen Möglichkeiten innerhalb von drei Monaten keine Heilungstendenz zeigt bzw. nicht innerhalb eines Jahres abgeheilt ist [4]. Bei ausgeprägter Therapieresistenz nimmt der Anteil polyätiologischer Ursachen der Ulzerationen deutlich zu. Bis zu $70 \%$ der Betroffenen weisen dann zusätzlich zu einer CVI eine pAVK auf, gefolgt von einer Dreierkombination aus venöser, arterieller und vaskulitischer Ätiologie [17]. 


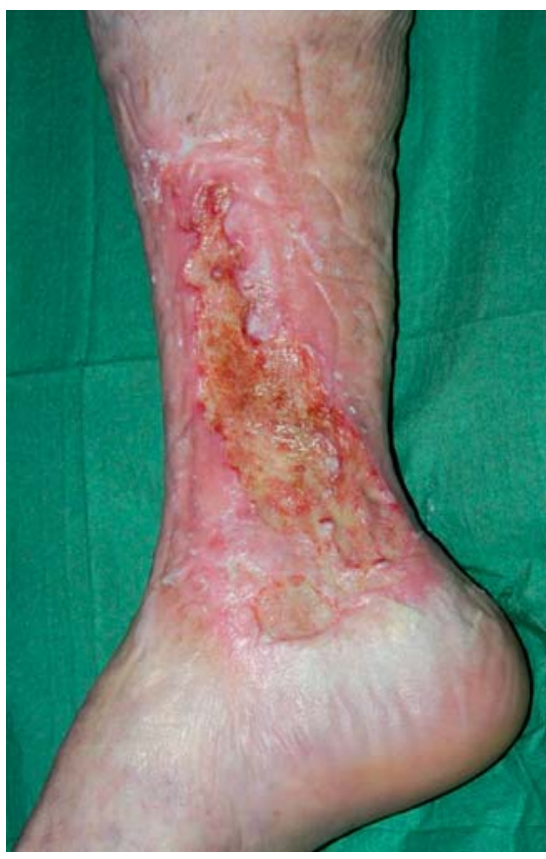

Abb. 1 Ulcus cruris venosum.

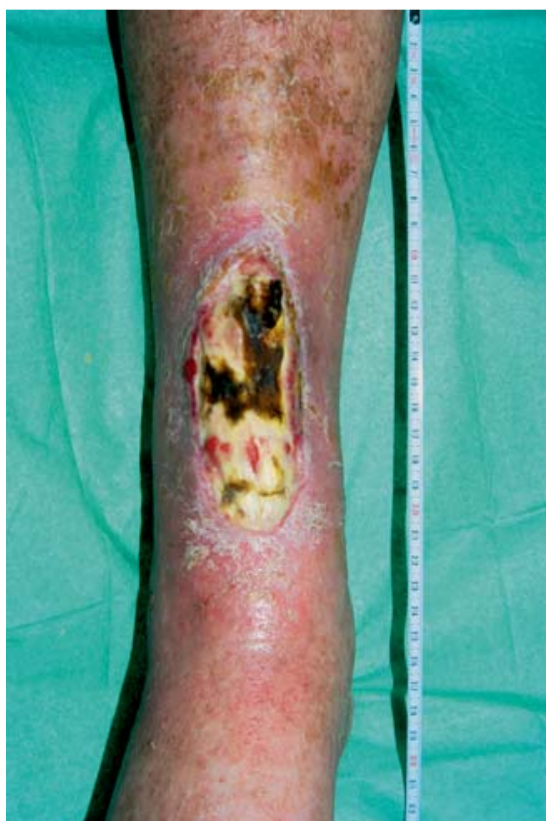

Abb. 2 Ulcus cruris arteriosum.

\section{Differenzialdiagnose}

Neben den genannten Ursachen können auch andere Erkrankungen zum Auftreten eines Ulcus cruris führen. Unter anderem ist dann an Vaskulitiden, Malignome, Stoffwechselerkrankungen oder Infektionen zu denken ( $\bullet$ Tab. 1, $\odot$ Abb. 3, 4).

\section{Klinik und klinische Untersuchung}

$\nabla$

Das Ulcus cruris venosum als schwerste Form der CVI ist durch einen Substanzdefekt in pathologisch verändertem Gewebe des Unterschenkels charakterisiert [4]. Das klinische Bild reicht von einer kleinen, umschriebenen Ulzeration im Bereich des medialen Malleolus innerhalb trophisch veränderter Haut bis zu großen, die gesamte Zirkumferenz des Unterschenkels einnehmenden Ulzerationen mit ausgedehnter Dermatoliposklerose, Dermatoli-
Tab. 1 Differenzialdiagnose des Ulcus cruris (nach $[2,4]$ ).

\begin{tabular}{|c|c|}
\hline Vaskuläre Ursachen & $\begin{array}{l}\text { CVI } \\
\text { PAVK isoliert oder in Kombination mit } \\
\text { CVI } \\
\text { Lymphabflussstörungen (Lymphödem, } \\
\text { Dysplasie) }\end{array}$ \\
\hline $\begin{array}{l}\text { Vaskulopathien/ } \\
\text { Mikrozirkulationsstörungen }\end{array}$ & $\begin{array}{l}\text { Diabetische Mikroangiopathie } \\
\text { Ulcus hypertonicum } \\
\text { Livedovaskulopathie } \\
\text { Embolien } \\
\text { Kalziphylaxie }\end{array}$ \\
\hline Infektionen & $\begin{array}{l}\text { Bakterien, Mykosen } \\
\text { Viren, Parasiten }\end{array}$ \\
\hline Vaskulitiden & $\begin{array}{l}\text { Leukozytoklastische Vaskulitis } \\
\text { Vaskulitiden i. R. von Autoimmun- } \\
\text { erkrankungen (Kollagenosen, rheuma- } \\
\text { toider Arthritis u. a.) } \\
\text { Polyarteritis nodosa }\end{array}$ \\
\hline $\begin{array}{l}\text { Hämatologische und } \\
\text { myeloproliferative } \\
\text { Erkrankungen }\end{array}$ & $\begin{array}{l}\text { Sichelzellanämie, Thalassämie u. a. } \\
\text { Polycytämia vera, Thrombozythämie, } \\
\text { M. Werlhof }\end{array}$ \\
\hline Neuropathische Ursachen & $\begin{array}{l}\text { Peripher: Diabetes mellitus, Alkohol, } \\
\text { Medikamente } \\
\text { Zentral: Multiple Sklerose, u. a. }\end{array}$ \\
\hline Neoplasien & $\begin{array}{l}\text { Basalzell-, Plattenepithelkarzinom, } \\
\text { Melanom, Lymphom, Sarkom }\end{array}$ \\
\hline Metabolische Ursachen & $\begin{array}{l}\text { Diabetes mellitus, Amyloidose, Gicht, } \\
\text { Porphyrien u. a. }\end{array}$ \\
\hline Dermatosen & $\begin{array}{l}\text { Pyoderma gangraenosum, Necrobiosis } \\
\text { lipoidica, Sarkoidose u. a. }\end{array}$ \\
\hline Medikamente & Marcumar, Hydroxyurea, MTX u. a. \\
\hline Exogene Ursachen & $\begin{array}{l}\text { Wärme, Kälte, ionisierende Strahlung, } \\
\text { Artefakte, chemische Noxen }\end{array}$ \\
\hline
\end{tabular}

pofasziosklerose, Nekrose von Faszien- und Sehnenanteilen und arthrogenem Stauungssyndrom. In der täglichen Praxis haben sich die Einteilungen nach Widmer [19] und Hach [6,7] bewährt. Insbesondere die Einteilung nach Hach eignet sich aufgrund der relevanten Gewebsveränderungen für die operative Ulkustherapie ( $\odot$ Tab. 2).

Bereits die klinische Untersuchung und die Erhebung des Lokalbefundes mit Beurteilung des Ulkus (Lokalisation, Größe, Tiefe und Rand) und der Ulkusumgebung (Rötung, Sklerose, Ekzem, Ödem etc.) sowie des Venen- und Pulsstatus können entscheidende Hinweise bzgl. der Genese des Ulkus geben. Typischerweise sind venöse Ulzera meist am Innenknöchel lokalisiert, während Ulzerationen am lateralen Unterschenkel oder prätibial ein Hinweis für eine (zusätzliche) pAVK sein können. Bei klinisch unklarem oder suspektem Befund sollten Probebiopsien zur weiteren Abklärung erfolgen. Bei der körperlichen Untersuchung sollte zumindest orientierend ein neurologischer und orthopädischer Status der unteren Extremitäten erfolgen. Hierdurch sind prognostisch und therapeutisch entscheidende Faktoren, wie Sensibilitätsstörungen im Rahmen von Polyneuropathien, und Gelenkveränderungen, wie das arthrogene Stauungssyndrom, zu erkennen $(\bullet$ Abb. 5).

\section{Apparative Diagnostik \\ $\nabla$}

Die apparative Diagnostik dient dazu, einen aussagekräftigen Befund über den vorliegenden Gefäßstatus zu bekommen. Sowohl die arterielle als auch venöse Situation sind hier von entscheidender Bedeutung. Basistherapeutisch sollte eine direktionale 


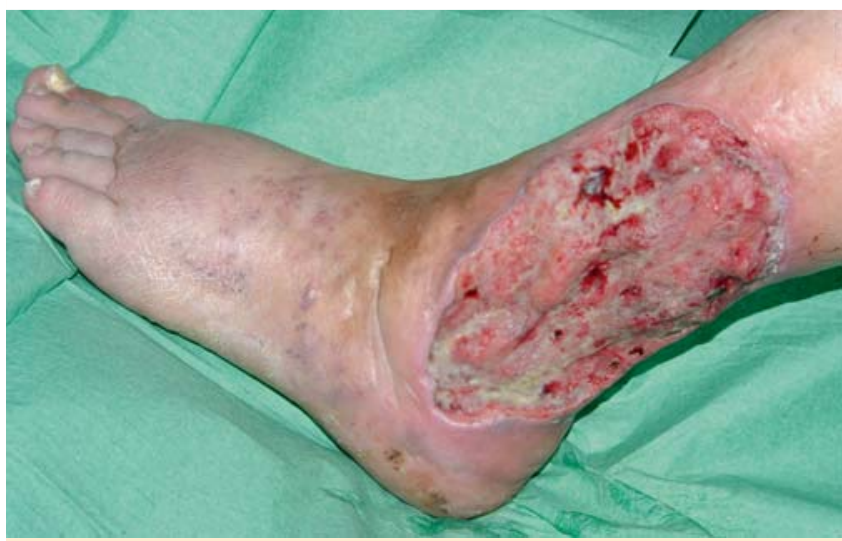

Abb. 3 Plattenepithelkarzinom bei Ulcus cruris, sog. Narben-Karzinom.

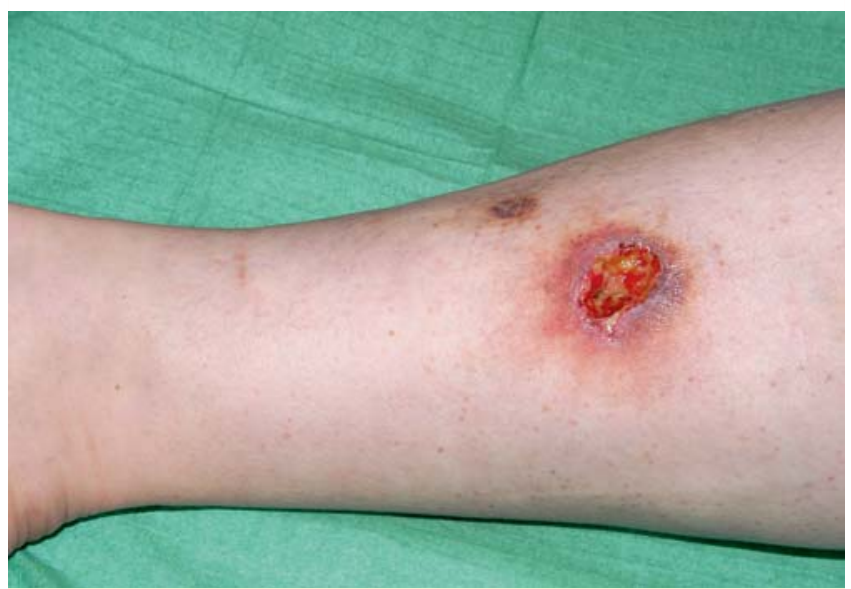

Abb. 4 Pyoderma gangraenosum.

Doppler-Sonografie der Beinvenen und -arterien erfolgen. Des Weiteren muss die Ermittlung des systolischen Knöchelarteriendruckes in Korrelation zu den Brachialarterien, ggf. mit Darstellung der Doppler-Signalkurve, erfolgen. Ist diese auffällig, die Anamnese hinsichtlich einer pAVK verdächtig oder sind die Fußpulse nicht oder nur schwach tastbar, sollte eine (farbkodierte) Duplexsonografie der Beinarterien erfolgen. Bestätigt sich der Verdacht auf das Vorliegen einer relevanten arteriellen Komponente, sollten sich weitere Untersuchungen anschließen. In der Regel wird dann eine digitale Subtraktionsangiografie (DSA) oder eine Angio-MRT notwendig, da sie die Voraussetzungen für

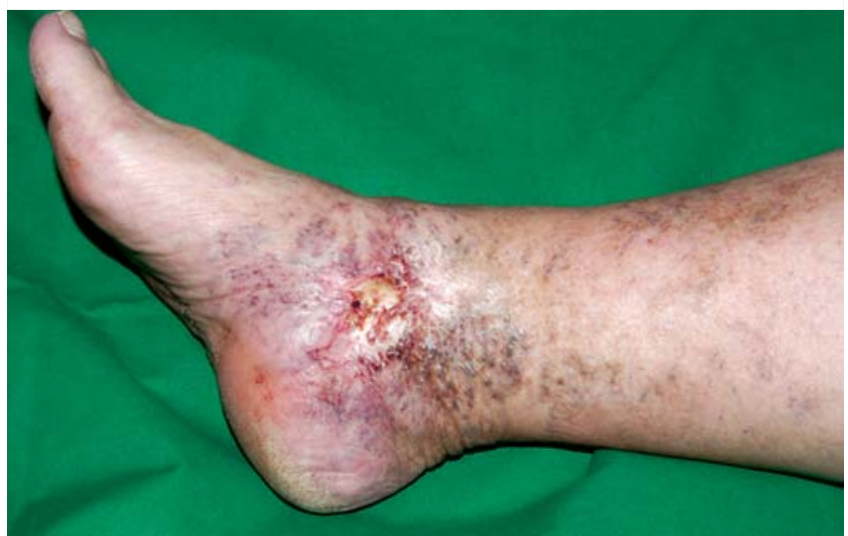

Abb. 5 Exulzerierte Capillaritis alba.

gegebenenfalls folgende invasive therapeutische Maßnahmen (PTA, Stent-Implantation, Bypass-OP etc.) darstellen. Hinsichtlich der venösen Situation können funktionelle Untersuchungen wie die Lichtreflexionsrheografie/Fotoplethysmografie (mit und ohne Tourniquet) weitere wertvolle Informationen liefern. Je nach Befund und geplantem Eingriff können auch Untersuchungen der erweiterten Diagnostik notwendig werden. Hier sind in erster Linie die (farbkodierte) Duplexsonografie des Venensystems sowie die (Press-)Phlebografie zu nennen.

\section{Präoperative Maßnahmen}

Um ein möglichst gutes und stabiles postoperatives Ergebnis zu erzielen, ist es notwendig, potenzielle Komplikationsquellen bereits vor dem geplanten Eingriff zu behandeln. Insbesondere beim therapieresistenten Ulcus cruris liegen häufig Unterschenkelödeme, periläsionale Ekzeme oder eine bakterielle Infektion/ Besiedelung vor. Das Ulkus selbst sollte durch eine adäquate Wundkonditionierung vorbereitet werden ( $\bullet$ Tab. 3, $\bullet$ Abb. 6). Nicht nur die präoperative Behandlung möglicher Komplikationsquellen ist erforderlich, sondern auch die Durchführung notwendiger prophylaktischer Maßnahmen. Nicht selten bestehen bei Patienten mit therapieresistentem Ulkus relevante TypIV-Sensibilisierungen. Diese sollten, soweit nicht bereits bekannt, mittels Epikutantest vor operativen Maßnahmen abgeklärt werden. Hinsichtlich bestehender Allergien, die narkose- oder operationsbedingt zu erwarten sind, wie z. B. gegenüber Lokalanästhetika, Narkotika oder Latex, sollten ebenfalls geeignete allergologi-

\begin{tabular}{|c|c|c|}
\hline Stadium & CVII-III nach Widmer & CVII-IV nach Hach \\
\hline I & Corona phlebectatica, Phlebödem & Keine Gewebssklerose \\
\hline II & $\begin{array}{l}\text { Trophische Hautveränderungen } \\
\text { 1. Capillaritis alba/Atrophie blanche } \\
\text { 2. Purpura jaune d'ocre } \\
\text { 3. Stauungsdermatitis } \\
\text { 4. Dermatoliposklerose } \\
\text { 5. Akrodermatitis Mali }\end{array}$ & Dermatoliposklerose \\
\hline III & $\begin{array}{l}\text { Ulcus cruris } \\
\text { 1. abgeheilt } \\
\text { 2. floride }\end{array}$ & Dermatolipofasciosclerosis regionalis \\
\hline IV & & $\begin{array}{l}\text { Sklerotische Gewebeveränderungen der Haut, des } \\
\text { Subkutangewebes und der Faszie zirkulär am Unter- } \\
\text { schenkel mit ausgedehnter Ulzeration - Dermato- } \\
\text { lipofasciosclerosis circularis }\end{array}$ \\
\hline
\end{tabular}

Tab. 2 Stadieneinteilung der CVI. 


\begin{tabular}{|lll|}
\hline Läsional & Fibrinöse Beläge und Krusten & Wundkonditionierung \\
& Nekrosen & - Débridement \\
& Pathogene Keimbesiedelungen/Infektionen & - Stadiengerechte (antiseptische) Lokaltherapie \\
\hline Peripher & Ekzeme (z. B.mikrobiell, kontaktallergisch, & Adäquate Lokaltherapie \\
& irritativ, stauungsbedingt) & Kompressionstherapie \\
Ulkusrand/ & Mazeration & Allergenkarenz \\
-umgebung & Infektion & Wundrandprotektion \\
& Malnutrition: & Systemische Antibiose \\
\hline Kausal & Unterschenkelödeme (venös bedingt) & Kompressionstherapie \\
& Minderperfusion (arteriell bedingt) & Diuretika \\
\hline \multirow{2}{*}{ Systemisch } & Infektionen (z. B. Erysipel) & PTA, Stent, Bypass, Prostaglandine \\
& Anämie & Systemische Antibiose \\
& Allergien & Transfusion \\
& Thrombosen & Allergenkarenz, Prämedikation \\
\hline
\end{tabular}

Tab. 3 Präoperative Komplikationsquellen und Interventionsmöglichkeiten.

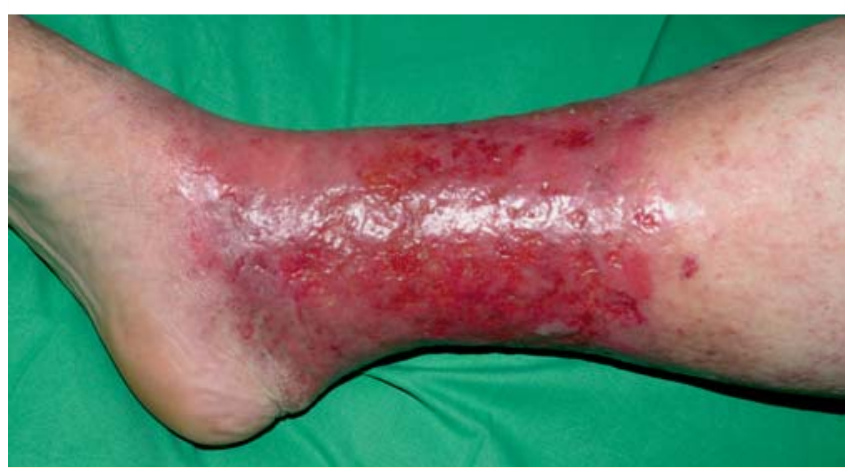

Abb. 6 Allergisches Kontaktekzem, Nachweis einer Typ-IV-Sensibilisierung gegen Refobacin.

sche (Ausweich-)Testungen oder entsprechende Prämedikationen erfolgen. Selbstverständlich ist bei der Auswahl der am Patienten angewendeten Substanzen auf eine strikte Allergenkarenz zu achten. Bei Infektionszeichen sollte eine systemische Antibiose, möglichst nach Antibiogramm, erfolgen. Bereits bei pathogener Keimbesiedelung ist zumindest eine perioperative antibiotische Therapie als „single shot“, z. B. mit Cefuroxim, empfehlenswert. Gerade bei größeren Eingriffen sollte eine perioperative Prophylaxe erfolgen, wobei hier auch die Bereitstellung von Erytrozytenkonzentraten sinnvoll ist. Zusätzlich sollte eine risikoadaptierte Thromboseprophylaxe mit niedermolekularem Heparin gegeben werden [16].

Um unrealistische Erwartungen von Seiten des Patienten auszuräumen und eine möglichst gute Compliance zu erreichen, sollte die Operationsaufklärung möglichst ausführlich und umfassend erfolgen und auch das notwendige postoperative Verhalten des Patienten, wie z.B. die Bettruhe nach Spalthaut-Transplantation, berücksichtigen.

\section{Operative Therapie}

Ist die Indikation zur operativen Ulkusbehandlung gegeben (siehe Definition des therapieresistenten Ulcus cruris), ist die Auswahl des entsprechenden Operationsverfahrens entscheidend. Neben dem Alter und dem Allgemeinzustand des Patienten ist die Berücksichtigung der ätiopathologisch zugrunde liegenden Ursachen des Ulkus bei der Festlegung des operativen Prozederes maßgebend. Im Wesentlichen basieren die Konzepte der operativen Sanierung des Ulcus cruris venosum auf drei therapeutischen Ansätzen:
- Eliminierung insuffizienter epi- und transfaszialer Venenabschnitte

- Lokale Verfahren/Ulkuschirurgie: Débridement, Ulkusausschneidung, tangentiale Abtragung der Dermatoliposklerose/ Shave-Therapie

- Techniken unter Einbeziehung der kruralen Faszie: paratibiale Fasziotomie, Fasziektomie

Die Rekonstruktion und Transplantation von Venenklappen im tiefen Venensystem, welche in den DGP-Leitlinien [4] als vierter Ansatz genannt wird, ist nur selten sinnvoll und nur wenigen spezialisierten Gefäßzentren vorbehalten.

\section{Eliminierung insuffizienter epi- und transfaszialer Venenabschnitte}

Phlebochirurgie und endoluminale Okklusionsverfahren

Ziel dieser Verfahren ist es, den pathologischen Reflux, der durch die vorliegende Varikose verursacht wird, auszuschalten. Diese Elimination kann vor oder parallel zum ulkuschirurgischen Eingriff erfolgen und führt zur Verbesserung oder idealerweise zur Normalisierung der venösen Hämodynamik $[8,10]$.

Indikation zur Ausschaltung insuffizienter epi- und transfaszialer Venenabschnitte ist die primäre Varikose, die sekundäre Varikose beim PTS (nicht bei Leitveneninsuffizienz) und die Rezidiv-Varikose. Jahrzehntelang galt die Entfernung von Stammvarizen mittels invaginierenden Strippings nach Krossektomie und/oder Seitenastvarizen inklusive insuffizienter Perforanzvenen als Standardverfahren. Mittlerweile stellen jedoch die endoluminalen Okklusionsverfahren mit Verzicht auf eine Krossektomie eine teilweie adäquate und gute Alternative dar. Verfahren, die sich in dieser Technik bewährt haben, sind:

- Schaumsklerosierung

- Endoluminale Lasertherapie (ELT)

- Radiofrequenzobliteration $\left(\right.$ VNUS $\left.^{\circledR}\right)$

Bei der Schaumsklerosierung wird ein aufgeschäumtes detergenzienartiges Sklerodierungsmittel, in der Regel Polidocanol, welches durch verschiedene Techniken in einen feinblasigen Schaum umgewandelt wird, nach Hochlagerung des entsprechenden Beines und möglichst duplexkontrollierter Punktion (mindestens $10 \mathrm{~cm}$ von der Krosse entfernt) in die insuffiziente Vene eingebracht. Im Anschluss verbessert eine konsequente Kompressionsbehandlung das Ergebnis. Die endoluminale Lasertherapie und die Radiofrequenzobliteration (VNUS ${ }^{\circledR}$ ) sind bzgl. der Technik ähnlich. Der wesentliche Unterschied besteht in der Energiequelle, die zum Verschluss der Vene benötigt wird. Bei beiden Verfahren wird nach duplexkontrollierter Punktion der Vene am distalen Insuffizienzpunkt der Lichtleiter bzw. der Wärmeenergie abgebende Katheter max. $2 \mathrm{~cm}$ vom Mündungspunkt der Vena sa- 
phena magna oder parva entfernt positioniert. Nach Tumeszenzanästhesie des gesamten Venenbetts werden anschließend entweder unter kontinuierlichem oder fraktioniertem Zurückziehen die entsprechenden Energiequanten endoluminal abgegeben. Dies führt dann zur Obliteration und später zur Fibrosierung des Gefäßes.

\section{Perforansvenenchirurgie}

Insofern die Indikation zur Perforantenentfernung gegeben ist, sollte man die endoskopische subfasziale Diszision von Perforansvenen (ESDP) aufgrund der geringeren postoperativen Komplikationsrate bei Patienten mit fortgeschrittener CVI dem transkutanen Zugang vorziehen $[16,18]$. Indiziert ist die ESDP bei schweren trophischen Störungen im Bereich ausgeprägter insuffizienter Perforansvenen. Sie wird heute seltener und gezielter eingesetzt [3].

\section{Ulkuschirurgie}

Lokale operative Maßnahmen am Ulkus sind dann sinnvoll, wenn die konservative Therapie und varizeneliminierende Methoden nicht erfolgreich waren oder das Ulkus (die Ulzera) für einen zeitlich vertretbaren Zeitraum zu großflächig ist (sind) [10]. Das Spektrum der zur Verfügung stehenden Techniken umfasst sowohl symptomatische Verfahren, die sich ausschließlich suprafaszial auf die Abtragung der Dermatoliposklerose, fibrotischer Beläge und/oder Nekrosen beschränken, als auch invasivere Maßnahmen, die unter Berücksichtigung des kausalen Therapieansatzes die krurale Faszie einbeziehen, wie die En-bloc-Resektion des Ulkus nach Homans, bei der der betroffene Faszienteil in die Resektion einbezogen wird.

\section{Wunddébridement}

Das Wunddébridement stellt aufgrund seiner relativ einfachen und risikoarmen Durchführbarkeit den Standard der suprafaszialen Ulkuschirurgie dar. Der Eingriff kann meist in Lokalanästhesie durchgeführt werden und ist nicht zuletzt daher auch für ältere Menschen geeignet. Daneben zeigt das Débridement eine hohe Effektivität und kann durch die Beseitigung heilungsbehindernder Krusten, Beläge oder Nekrosen ein aggressives Wundmilieu beseitigen und die Voraussetzung schaffen, dass eine Wundgrundgranulation (wieder) einsetzt. Im optimalen Fall kann das Débridement, ggf. wiederholt durchgeführt, zusammen mit konservativen Maßnahmen zur Abheilung des Ulkus führen. Des Weiteren stellt die mechanische Wundreinigung nahezu immer die Voraussetzung für den primären operativen Ulkusverschluss mittels Spalthaut-Transplantation ( $\bullet$ Abb. 7) dar.

Die Ulkus-Exzision als totales Débridement ist indiziert bei umschriebenen, therapieresistenten Ulzera mit lokalisierter Dermatolipo(faszio)sklerose oder kalzifizierter Sklerose, die eine ShaveTherapie wenig erfolgreich scheinen lassen ( $\bullet$ Abb. 8).

\section{Spalthaut-Transplantation}

Die Spalthaut-Transplantation ist die suffizienteste und schnellste Methode, um einen Defektverschluss des Ulkus zu erreichen. Um die Wahrscheinlichkeit für eine zügige Einheilung und einen dauerhaften und stabilen postoperativen Befund zu optimieren, sind jedoch einige Voraussetzungen von Bedeutung. Bei einer direkten Spalthaut-Transplantation, z.B. im Rahmen eines kombinierten Vorgehens mit Ulkus-Exzision oder tangentialer Liposkleroseabtragung, sollte ein ansprechender Wundgrund mit ausreichender Perfusion vorliegen. Bei zweizeitigem Vorgehen nach granulationsfördernder Konditionierung mit entsprechen-

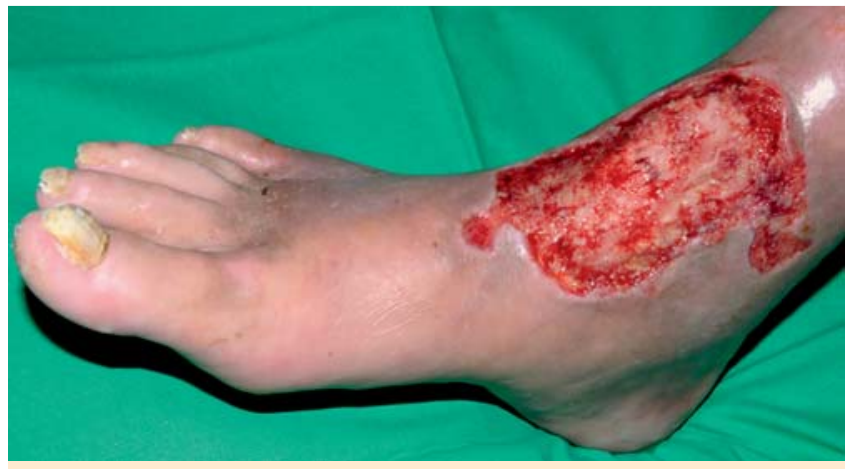

Abb. 7 Ulcus cruris, direkt postoperativ nach Débridement.

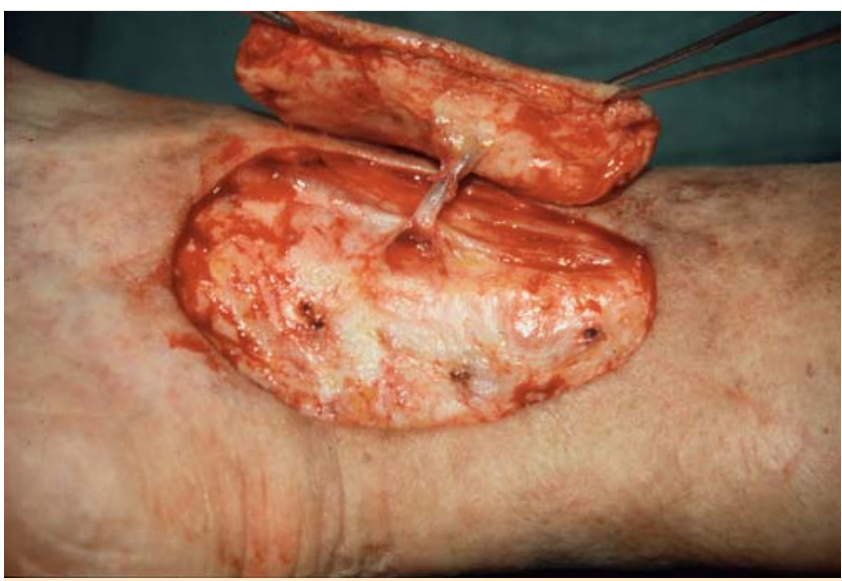

Abb. 8 Ulkus-Exzision (mit insuffizienter Perforansvene).

den Wundauflagen oder einer Vakuum-Versiegelung ist eine homogene, möglichst flächig ins Hautniveau reichende Granulation notwendig.

\section{Praktisches operatives Vorgehen}

In Abhängigkeit von Ulkusgröße, Lokalisation, Schmerzempfinden und Konstitution des Patienten kann der Eingriff meist in Lokalanästhesie erfolgen. Eine topische Vorbehandlung mit lidocain-/prilocainhaltigen Cremes und Gels (z.B. EMLA ${ }^{\circledR}$ ) kann die erforderliche Menge Lokalanästhetikums zur Infiltrationsanästhesie verringern. Auch die Tumeszenz-Anästhesie oder regional-anästhetische Verfahren können angewendet werden. Bezüglich der Entnahmestelle bietet sich in der Regel die laterale Oberschenkelseite an. Die Spalthautentnahme erfolgt idealerweise mit einem Elektro- oder Akku-Dermatom (Dicke je nach Anpressdruck und Hautbeschaffenheit 0,2 bis $0,4 \mathrm{~mm}$ ). Anschließend wird die entnommene Spalthaut durch kleine Walzmesser eines Mesh-Dermatoms mit feinsten Schnitten versehen und so in ein Maschengitter (Meshgraft) umgewandelt. In der Regel reicht ein Mesh-Verhältnis von 1:1,5 aus, um selbst große Defekte zu verschließen. Vorteil dieser Technik ist zum einen, dass durch die Oberflächenvergrößerung ca. ein Drittel weniger Spalthaut entnommen werden muss, und zum anderen, dass eine ausreichende Drainage durch die Gitterstruktur gewährleistet ist. Dies verhindert die Unterblutung des Transplantates und den Aufstau von Wundsekret. Bei der Transplantation des Meshgrafts ist zu beachten, dass dieses möglichst genau in den Defekt eingepasst wird und unter Nachahmung der physiologischen Spannung mittels Einzelknopf-/fortlaufender Naht oder Metallklammern fixiert wird. Um eine Abhebung oder Verschie- 


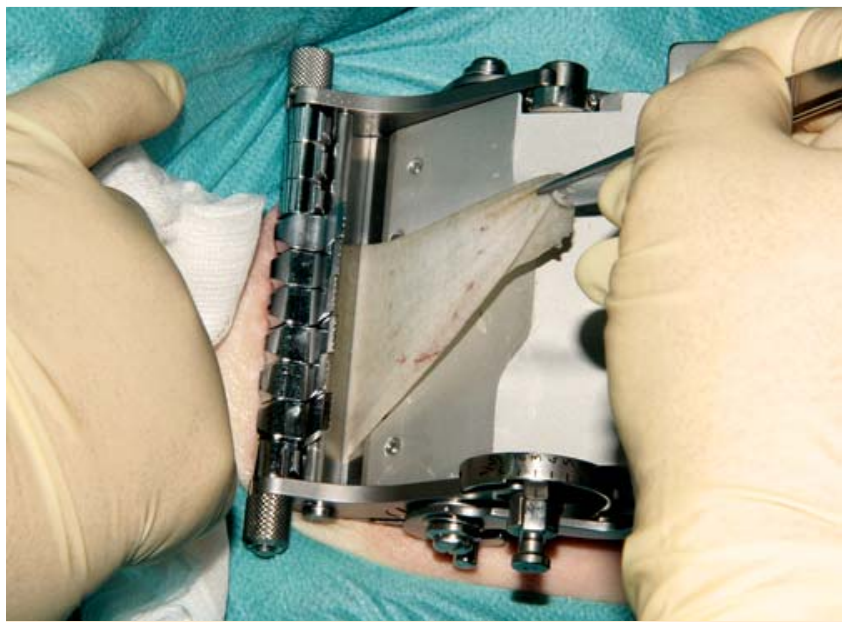

Abb.9 Spalthautentnahme mit Akku-Dermatom von der lateralen Oberschenkelseite (Dicke $0,3 \mathrm{~mm}$ ).

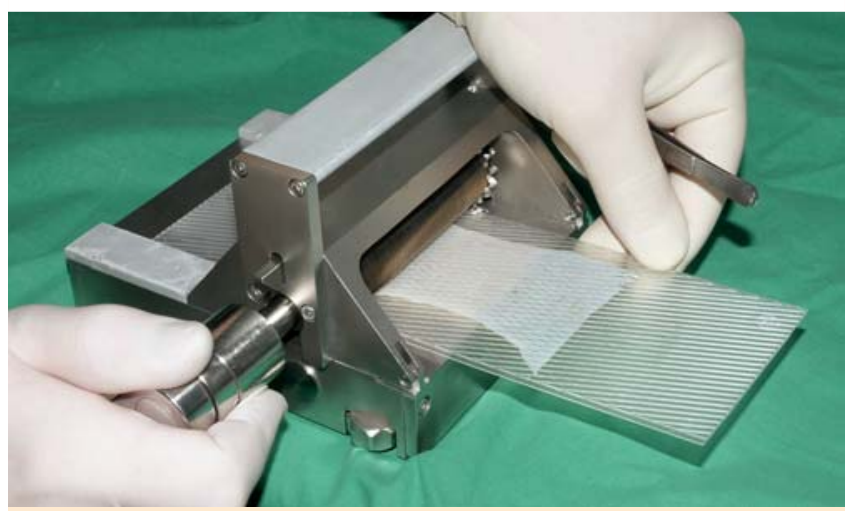

Abb. 10 Anfertigung eines Meshgrafts (Verhältnis der Transplantatvergrößerung 1:1,5).

bung des Transplantates zu verhindern, kann insbesondere bei malleolusnahen Lokalisationen eine zusätzliche Fibrinklebung erfolgen. Hierbei wird der flüssige Zweikomponentenkleber mittels stumpfer Kanüle zwischen Wundgrund und Transplantat eingebracht und gleichmäßig verteilt. Zum Schutz des Transplantates eignen sich Fett- oder Silikongazen. Die notwendige postoperative Kompression, die sich an der Gefäßsituation orientieren muss, kann mittels steriler Schaumstoffplatten und Kompressen zusätzlich fokussiert werden. Der erste Verbandswechsel erfolgt zwischen dem zweiten und vierten postoperativen Tag. Je nach Infektionsrisiko muss eine peri- bzw. postoperative prophylaktische Antibiotikagabe erwogen werden ( $\bullet$ Abb. 9-12).

\section{Tangentiale Dermatoliposklerose-Abtragung/}

\section{Shave-Therapie}

Die tangentiale Dermatoliposklerose-Abtragung, auch ShaveTherapie genannt, stellt insbesondere bei größerflächigen und länger bestehenden Ulzera, die in der Regel eine mehr oder weniger ausgeprägte Dermatolipo(faszio)sklerose aufweisen, das Verfahren der Wahl dar. Die gleichzeitige oder vorausgehende Sanierung insuffizienter extrafaszialer Venen ist zwar keine unmittelbare Voraussetzung der Shave-Therapie, erhöht jedoch die Wahrscheinlichkeit eines stabilen postoperativen Befundes.

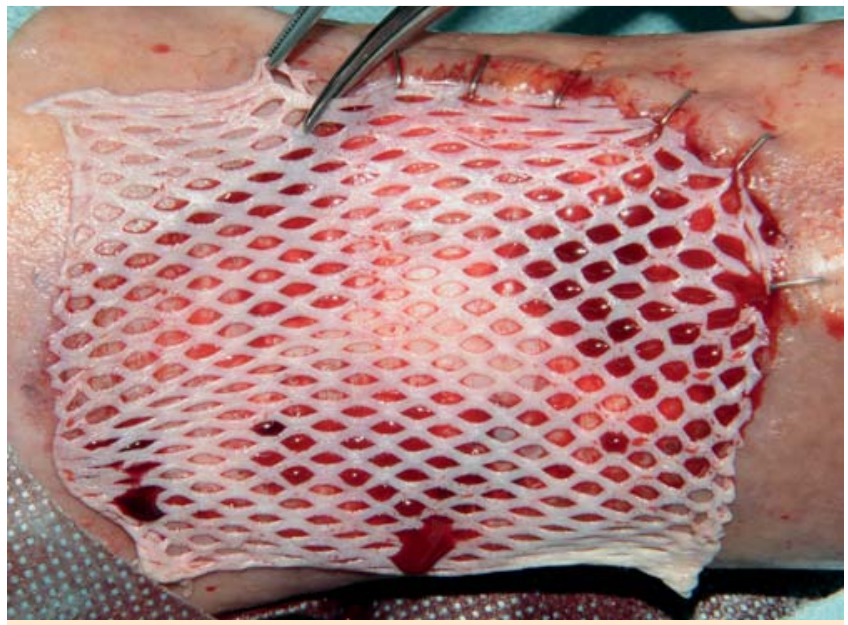

Abb. 11 Spalthaut-Transplantation (Einpassung und Fixation mittels Klammerung).

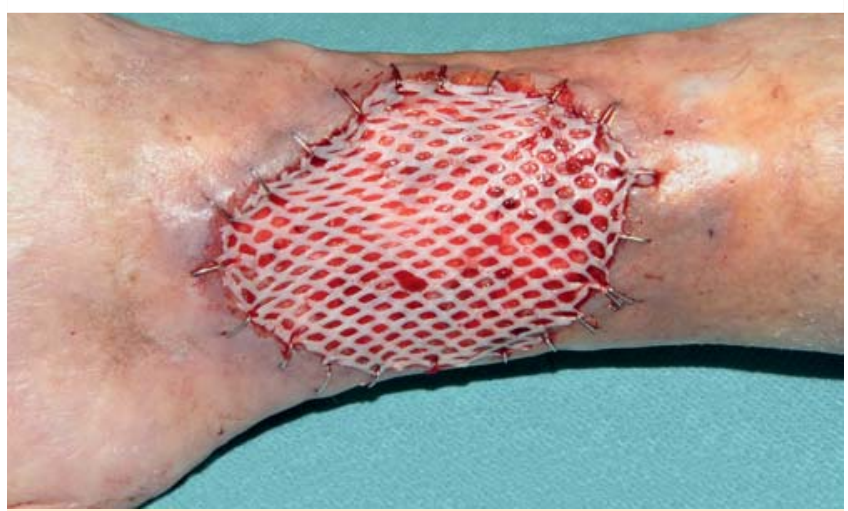

Abb. 12 Direkter postoperativer Befund nach erfolgter Spalthaut-Transplantation.

\section{Praktisches operatives Vorgehen}

Bei der tangentialen Dermatoliposklerose-Abtragung/ShaveTherapie handelt es sich um eine sequenzielle Flachexzisionstechnik, bei der manuell mittels Hand-Dermatom/SchinkelDermatom oder maschinell mit dem Elektro-/Akku-Dermatom tangential, schichtweise die oberen dermatoliposklerotisch veränderten Gewebsschichten des Ulkus und der Ulkusumgebung abgetragen werden. Das Ausmaß der Abtragung sollte soweit erfolgen, bis palpatorisch ein weicheres und besser perfundiertes Gewebe zu erkennen ist. Insuffiziente Venen, die durch dieses Verfahren mit erfasst werden, werden legiert, was zusätzlich zur Prognoseverbesserung beiträgt. Nach Beendigung der Abtragung kann die Wunde entweder direkt oder nach ausreichender granulationsfördernder Lokalbehandlung durch geeignete Wundauflagen oder Vakuumversiegelung (V.A.C.-Therapie ${ }^{\circledR}$ ) mittels Spalthaut-Transplantation in zweiter Sitzung verschlossen werden. Vorteile der Shave-Therapie sind höhere Einheilraten, kürzere Heilungszeiten und eine bessere Langzeitprognose $[1,8,15]$ (๑ Abb. 13).

\section{Paratibiale Fasziotomie}

Das Verfahren, von Hach 1983 eingeführt, sorgt für eine sofortige Herabsetzung des pathologisch erhöhten subfaszialen Gewebsdrucks, wobei insbesondere tiefe mediale Muskelkompartimente entlastet werden. Zudem kommt es zu einer anhaltenden Reaktivierung der lokalen Mikrozirkulation [11]. In den letzten Jahren 


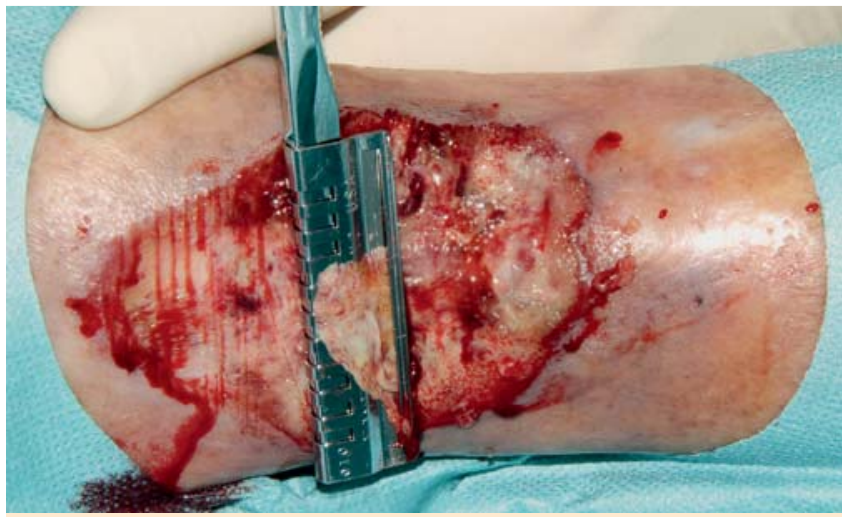

Abb. 13 Shave-Therapie, tangentiale Abtragung der Liposklerose mit Handdermatom.

wird die PTF seltener durchgeführt, was daran liegt, dass die Indikation zunehmend strenger und exakter gestellt wurde. Als Voraussetzung zur Durchführung einer paratibialen Fasziotomie (PTF) wird heute der Nachweis von morphologischen und funktionellen Veränderungen eines chronischen venösen Kompartmentsyndroms gefordert [4].

\section{Praktisches operatives Vorgehen}

In Allgemeinnarkose oder Spinalanästhesie erfolgt der Zugang mittels medio-paratibialer Hautinzision. Diese ist ausnahmslos extraläsional im proximalen bis mittleren Unterschenkeldrittel zu setzen. Nach Präparation wird die krurale Faszie an ihrem ossären Ansatz längs inzidiert. Anschließend werden durch kaudales, subfasziales Vorschieben eines Dissektionsspatels die Perforansvenen durchtrennt. Im Anschluss daran erfolgt in gleicher Weise die Spaltung der Fascia cruris mit dem Fasziotom nach Hach von proximal nach distal. Die unmittelbar auftretende Blutung, die auftritt, falls nicht in Blutleere operiert wird, ist durch Hochlagerung und manuelle Kompression problemlos zu kontrollieren. Vor dem stufenweisen Wundverschluss sollte eine subfasziale Redon-Drainage eingelegt werden. Unmittelbar postoperativ erfolgt die Anlage eines Kompressionsverbandes.

\section{Fasziektomie}

Die Fasziektomie, als radikalste Form der ulkuschirurgischen Maßnahmen, findet bei großflächigen, (nahezu) komplett zirkulären Ulzera (sog. Gamaschen-Ulkus) mit ausgeprägter Dermatolipofasziosklerose Anwendung. Insbesondere bei nekrotischen Sehnen- und/oder Faszienanteilen und kalzifizierter Fibrosklerose ist die Fasziektomie die chirurgische Therapie der Wahl $[4,9,15]$.

\section{Praktisches operatives Vorgehen}

Ebenfalls in Allgemeinnarkose oder Spinalanästhesie wird das gesamte betroffene Areal, welches das Ulkus, die Dermatoliposklerose und den entsprechenden Faszienanteil umfassen sollte, exzidiert. Der Eingriff sollte unter kontinuierlicher kontrollierter Blutstillung oder in Blutleere (z.B. mit pneumatischer Rollmanschette) erfolgen, da es zu stärkeren Blutungen kommen kann. Gegebenenfalls kann die Bereitstellung von Erythrozytenkonzentraten notwendig sein. Postoperativ schließt sich eine Wundkonditionierung an, die durch geeignete Wundauflagen oder Vakuumversiegelung (V.A.C.-Therapie ${ }^{\circledR}$ ) Voraussetzung für eine $a b-$ schließende Spalthaut-Transplantation ist ( $\bullet$ Abb. 14-18).

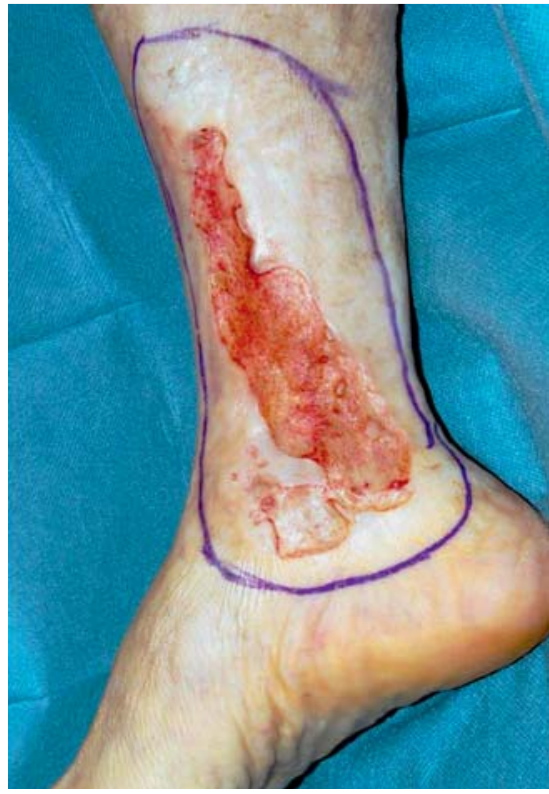

Abb. 14 Ulcus cruris, präoperativ mit Anzeichnung der Dermatoliposklerose.

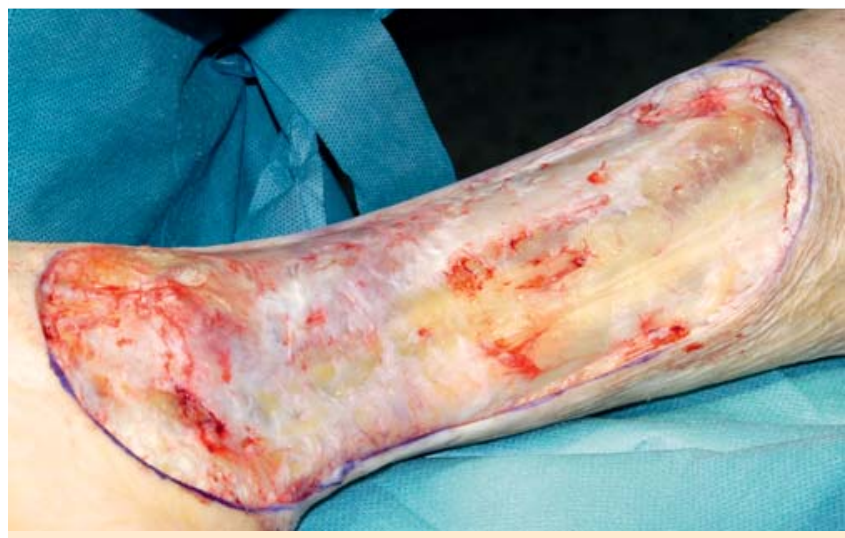

Abb. 15 Ulcus cruris, intraoperativ, Z.n. kompletter Fasziektomie.

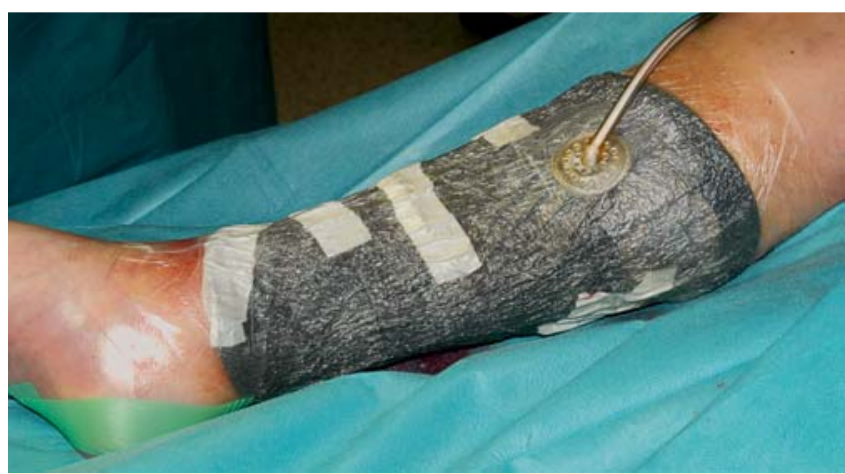

Abb. 16 Ulcus cruris, direkt postoperativ, Z. n. Anlage eines Vakuumverbandes (V.A.C.).

\section{Intra- und postoperative Komplikationen}

Neben den allgemeinen Operationsrisiken, wie bakterielle Infektionen, kann es bei den verschiedenen Operationsverfahren jeweils zu speziellen oder typischen Komplikationen kommen (๑ Tab.4). 


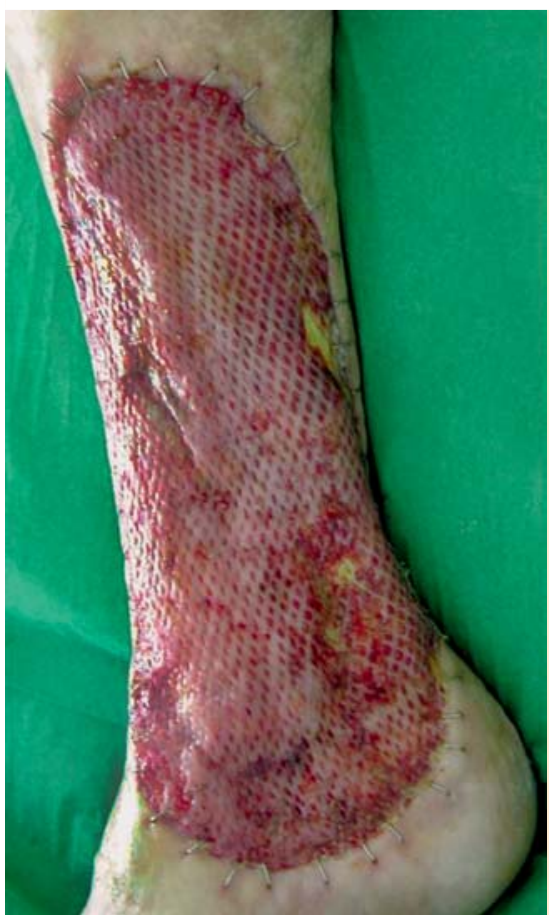

Abb. 17 Ulcus cruris, 6. postoperativer Tag nach anschließender Spalthaut-Transplantation.

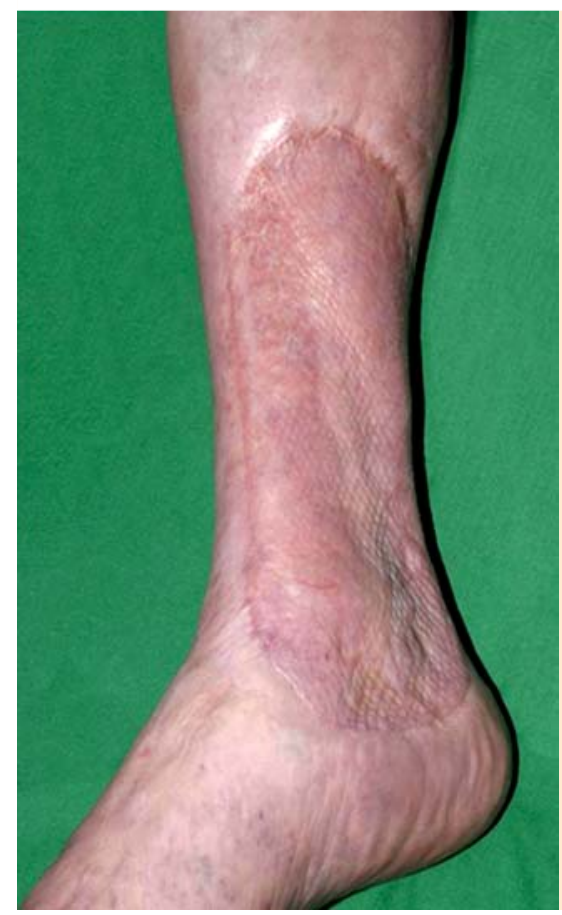

Abb. 18 Ulcus cruris, Befund nach 4 Monaten.

\section{Postoperative Therapie und Prophylaxe}

Voraussetzung für einen dauerhaften Therapieerfolg ist die konsequente Nachbehandlung. Hierzu gehören eine lebenslange, suffiziente medizinische Kompressionstherapie, in der Regel durch Kompressionsstrümpfe, die Fortführung der Entstauungsbehandlung durch apparative intermittierende Kompression und manuelle Lymphdrainage sowie eine krankengymnastische Beübung. Regelmäßige Kontrolluntersuchungen erhöhen die Qualität der postoperativen Therapie und Prophylaxe, da Fehlentwicklungen (z. B. insuffiziente Kompression), mögliche Gefährdungen (z.B. falsche oder fehlende Fußpflege, Tinea pedum) oder Rezidive frühzeitig erkannt und behandelt werden können.
Tab.4 Ulkuschirurgisches Verfahren - spezielle/typische Komplikationen.

\begin{tabular}{|ll|}
\hline $\begin{array}{l}\text { Endoskopische subfasziale Diszision } \\
\text { von Perforansvenen (ESDP) }\end{array}$ & $\begin{array}{l}\text { Persistierende Dysästhesien; } \\
\text { Ödemneigung; Rezidiv }\end{array}$ \\
\hline Spalthaut-Transplantation & $\begin{array}{l}\text { Partieller oder kompletter } \\
\text { Transplantatverlust }\end{array}$ \\
\hline $\begin{array}{l}\text { Tangentiale Dermatoliposklerose- } \\
\text { Abtragung }\end{array}$ & $\begin{array}{l}\text { Schädigung peripherer, } \\
\text { sensibler Nerven; } \\
\text { Shave-Therapie }\end{array}$ \\
\hline Paratibiale Fasziotomie & $\begin{array}{l}\text { Blutung/Nachblutung; } \\
\text { subfasziales Hämatom; } \\
\text { Dysästhesie (N. saphenus) }\end{array}$ \\
\hline Fasziektomie & $\begin{array}{l}\text { Blutung/Nachblutung; } \\
\text { Dysästhesie; schwierige Wund- } \\
\text { konditionierung }\end{array}$ \\
\hline
\end{tabular}

Nicht zuletzt trägt langfristig eine intensive Patientenschulung zu einem dauerhaften Erfolg und damit zu einer verbesserten Lebensqualität von Ulkus-Patienten bei [16].

Interessenkonflikt: Der Autor gibt an, dass kein Interessenkonflikt besteht.

\section{Abstract}

\section{Surgical Treatment of Leg Ulcers \\ $\nabla$}

Frequently the clinical picture of chronic leg ulcers poses a challenge in treatment. In spite of an adequate use of wound-dressings there is often a resistance to treatment. To transcend this problem it is necessary to exert a functional surgery of leg ulcers. Today there are numbers of established surgical methods available, which are symptomatic as well as causal. The decisive factor of a successful therapy is on one hand the correct indication and on the other hand the best selection and combination of the surgical procedure. Based on this fact it is essential to incorporate the causation of the leg ulcers. Essential for the durable prosperity of therapy is also the consistent after-treatment.

\section{Literatur}

1 Bechara FG, Stücker M, Altmeyer P et al. Shave-Therapie in der Behandlung des therapieresistenten Ulcus cruris. Vasomed 2005; 17: 88 - 92

2 Dissemond J, Körber A, Grabbe S. Differenzialdiagnosen des Ulcus cruris. JDDG 2006; 4: 627-634

3 Fischer R, Schwahn-Schreiber C, Sattler G et al. Die Indikation zur subfaszialen endoskopischen Perforantensanierung hat sich geändert. Phlebologie 2004; 33: 145-148

4 Gallenkemper G, Ehresmann U, Hermanns HJ et al. Leitlinie zur Diagnostik und Therapie des Ulcus cruris venosum. Phlebologie 2004; 33: $166-185$

5 Hach W, Hach-Wunderle V. Die Graduierung der chronisch venösen Insuffizienz. Gefäßchirurgie 2000; 5: 255-261

6 Hach W, Hach-Wunderle V. Neue Aspekte zum chronischen venösen Kompartmentsyndrom. Gefäßchirurgie 2001; 6: 164-169

7 Hermanns HJ. Operative Therapie des Ulcus cruris venosum. Gefäßchirurgie 2006; 11: $281-286$

8 Hermanns HJ, Gallenkemper G, Kanya S et al. Die Shave-Therapie im Konzept der operativen Behandlung des therapieresistenten Ulcus cruris venosum. Phlebologie 2005; 34: 209-215

9 Hermanns HJ, Schwahn-Schreiber C, Waldermann F. Stellenwert der operativen Verfahren in der Behandlung des Ulcus cruris venosum. Phlebologie 2006; 35: 199-203

10 Proebstle TM. Operative Ulkus-Therapie. Hautarzt 2003; 54: 379- 388

11 Proebstle TM, Pannier FM, Schuller-Petrovic S et al. Konsensus zur endovenösen Lasertherapie der Varikose. Phlebologie 2004; 33: 106 - 109 
12 Rabe E, Pannier-Fischer F, Schuldt K et al. Bonner Venenstudie der Deutschen Gesellschaft für Phlebologie zur Frage der Häufigkeit und Ausprägung von chronischen Venenkrankheiten in der städtischen und ländlichen Wohnbevölkerung. Phlebologie 2003; 32: 1-14

13 Sigala F, Hepp W, Menenakos V, Mommertz et al. Paratibiale Fasziotomie mit Perforansdissektion. Phlebologie 2007; 36: 260-264

14 Schmeller W, Gaber Y. Persistierendes Ulcus cruris und chronisches venöses Kompartmentsyndrom. Phlebologie 2001; 30: 75-80

15 Schmeller W, Schwahn-Schreiber C, Gaber Y. Langzeitergebnisse nach Shave-Therapie bzw. kruraler Fasziektomie bei persistierenden venösen Ulzera. Phlebologie 2006; 35: 89-91
16 Stein A, Hackert I. Lokale operative Ulkustherapie. Hautarzt 2007; 58: $781-796$

17 Stücker M, Harke K, Rudolph T et al. Zur Pathogenese des therapieresistenten Ulcus cruris. Hautarzt 2003; 54: 750 - 755

18 Sybrandy JE, van Gent WB, Pierik EG et al. Endoscopic versus open subfascial division of incompetent perforating veins in the treatment of venous leg ulceration: long-term follow-up. J Vasc Surg 2001; 29: $1028-1032$

19 Widmer LK, Stähelin HB, Nissen C et al. (Hrsg). Venen-, Arterienkrankheiten, koronare Herzkrankheit bei Berufstätigen. Prospektiv-epidemiologische Untersuchung Baseler Studie I-III 1959-1978. Bern: Huber, 1981: 69f 